\title{
Color Tuning Mechanism of Human Red, Green, and Blue Cone Pigments: SAC-CI Theoretical Study
}

\author{
Kazuhiro Fujimoto, ${ }^{1, \dagger}$ Jun-ya Hasegawa, ${ }^{1,2}$ and Hiroshi Nakatsujii, ${ }^{* 1,2}$ \\ ${ }^{1}$ Department of Synthetic Chemistry and Biological Chemistry, Graduate School of Engineering, \\ Kyoto University, Nishikyo-ku, Kyoto 615-8510 \\ ${ }^{2}$ Quantum Chemistry Research Institute (QCRI) and JST-CREST, Kyodai Katsura Venture Plaza, \\ 1-36 Goryou Oohara, Nishikyo-ku, Kyoto 615-8245
}

Received May 15, 2009; E-mail: h.nakatsuji@qcri.or.jp

\begin{abstract}
Human red (HR), green (HG), and blue (HB) cone pigments are responsible for human color vision, and their photoabsorption wavelengths spread uniquely over the three primary colors. These pigments, however, include only one common chromophore, retinal. Here, we report physical basis of the color tuning in human vision on the basis of SAC-CI calculations for excited states of the cone pigments. The dominant origin of the red-green-blue distinction lies in differences in electrostatic interactions between retinal and its surrounding proteins. Structural distortion effect of the retinal chromophore is important in human blue pigment. Detailed analysis on individual roles of amino acids within these proteins has clarified elaborate mechanisms of the color tuning, in accordance with previous mutagenesis experiments. Furthermore, the color tuning is regulated by amino acids at specific positions in the proteins, suggesting some genetic origins for the color tuning.
\end{abstract}

Molecular processes of human color vision start with photochemical reactions in visual cone pigments in retina. ${ }^{1-6}$ In human retina, there are three cone pigments, human blue $(\mathrm{HB})$, green $(\mathrm{HG})$, and red $(\mathrm{HR})$ and they are responsible for the color distinction. ${ }^{7}$ Absorption wavelengths of the $\mathrm{HB}, \mathrm{HG}$, and HR pigments are $414 \mathrm{~nm}(2.99 \mathrm{eV}),{ }^{3,8,9} 532 \mathrm{~nm}$ $(2.33 \mathrm{eV}),{ }^{3,8,10}$ and $563 \mathrm{~nm}(2.20 \mathrm{eV}),{ }^{3,8,10}$ respectively. After amino acid sequences of these pigments were identified, ${ }^{11}$ pioneering mutagenesis studies ${ }^{4,9,10,12-14}$ were conducted for clarifying biological origin of the color tuning, but physical mechanism of the color tuning has been a long-standing problem, which was difficult to solve solely by one-dimensional information such as homology or differences among the amino acid sequences. It is now possible to clarify basic physics behind the color tuning in visual pigments by applying modern electronic structure theories (for example, see ${ }^{15-19}$ and references therein). The present study helps to understand general features of the color tuning mechanism among the cone pigments of vertebrates and, at the same time, an origin of the color vision deficiency.

Regarding theoretical studies on absorption spectrum of retinal, starting with the quantum-mechanical/molecular-mechanical $(\mathrm{QM} / \mathrm{MM})$ studies in solutions, ${ }^{20,21}$ many studies were reported on the spectral tuning of the rod pigment, bacteriorhodopsin, and sensory rhodopsin II. ${ }^{15-19,22-34}$ In our knowledge, there is, however, only a CI-single level of study ${ }^{32}$ on human cone pigments. Semiquantitative calculations ${ }^{32}$ could not reproduce experimental absorption energies, so it was difficult to discuss the color tuning mechanism.

$\dagger$ Present address: Department of Chemistry, Graduate School of Science, Kyoto University, Sakyo-ku, Kyoto 606-8502
In this study, we theoretically determined structures of human visual pigments and clarified molecular mechanisms behind the color tuning in the three cone pigments. On the basis of the calculations for excited states of human cone pigments using symmetry adapted cluster-configuration interaction (SAC-CI) method, ${ }^{35-38}$ we clarified electronic structure of retinal protonated Schiff base (PSB) and interactions between PSB and the opsin environments. ${ }^{39}$ SAC-CI method has already been established as a reliable method for calculating excited states of small molecules to photobiological systems. ${ }^{40,41}$ The method was successfully applied to photosynthetic reaction centers ${ }^{42-44}$ and retinal proteins, such as $\mathrm{HB},{ }^{17}$ rhodopsin $(\mathrm{Rh})^{33,45}$ and the proton-pump system. ${ }^{33,45} \mathrm{We}$ also performed detailed analysis on individual roles of amino acids within these proteins, which has clarified elaborate mechanisms of the color tuning, in accordance with previous mutagenesis experiments. ${ }^{4,9,10,12}$ The present results have added new understanding based on three-dimensional information, such as molecular structures, electronic wave functions, and intermolecular interactions in the cone pigments. Furthermore, we report that the color tuning is regulated by amino acids at specific positions in the opsins, suggesting some genetic origins for the color tuning.

\section{Computational Details}

In the geometry optimizations, we started from the homology modeling structures (PDB: 1KPX, 1KPW, and 1KPN for HR, $\mathrm{HG}$, and $\mathrm{HB}$, respectively) ${ }^{46}$ as initial guesses. To improve the protonation states of the charged residues, we performed PoissonBoltzmann calculations ${ }^{47}$ by using MEAD (macroscopic electrostatics with atomic detail) program to determine local $\mathrm{p} K_{\mathrm{a}}$ of ionizable amino acids. In Table S1 in the Supporting Information 
(SI), we provided a list of protonation states adopted in the present study. In addition, the positions of the water molecules were also modified according to the X-ray structures of $\mathrm{Rh}^{48}$ (PDB: 1L9H). In the $\mathrm{HG}$ and $\mathrm{HR}$, the $\mathrm{Cl}^{-}$binding sites were introduced in the vicinity of amino acids at positions 181 and 197 on the basis of the experimental mutation results. ${ }^{13}$

Structures and excited states of the cone pigments were calculated using the hybrid QM/MM method. ${ }^{49}$ The QM/ MM program ${ }^{24,50}$ which we used in our previous studies was successfully applied to retinal proteins. ${ }^{17,24,33,45,50}$ We also implemented the QM/MM code $39,45,51$ into the development version of Gaussian03. ${ }^{52}$ Further details of the QM/MM method were given in the SI. In the geometry optimizations, density functional theory $\left(\mathrm{DFT}^{53}\right.$ ) with B3LYP functional ${ }^{54,55}$ was used for the QM region, and AMBER99 force field ${ }^{56}$ was used for the MM region. SAC-CI calculations were performed using local energy minimum structures obtained by the QM/MM calculations. MD calculations were performed in constructing the homology modeling structures ${ }^{46}$ which were used as the reference in this study. Statistical average by the MD sampling should be done in the next step. ${ }^{57}$

Regarding the QM/MM boundary, we used two models as shown in Figure 1. The RET model, that was used for analysis, consists of only retinal PSB chromophore. The active site (AS) model includes the counter ion residue and a neighboring water in addition to the RET model. The QM/MM boundary was at the $\mathrm{C}_{\beta}-\mathrm{C}_{\gamma}$ bond of lysine and the $\mathrm{C}_{\alpha}-\mathrm{C}_{\beta}$ bond of aspartate as shown in Figure 1 . The $\mathrm{C}_{\beta}$ of Lys and the $\mathrm{C}_{\alpha}$ of Asp were replaced by the $\mathrm{H}$ atoms.

In both B3LYP and SAC-CI calculations, a double- $\zeta$ polarization basis ${ }^{58}$ was used for the carbons of the retinal $\pi$-system, the $\mathrm{N}$ and $\mathrm{H}$ atoms of the PSB, and the $\mathrm{O}$ and $\mathrm{C}$ atoms of the carboxylate groups in the counter ion residue and water. Double- $\zeta$ sets $^{58}$ were used for the rest of the atoms. Furthermore, a single ptype anion function $(\alpha=0.059)^{58}$ was augmented on the $\mathrm{O}$ atoms of the counter ion group. This level of basis sets was sufficient for calculating absorption energies of the systems. ${ }^{17,33,45}$ In SAC-CI calculations, all valence orbitals were included in the active space. The core orbitals and corresponding virtual orbitals were treated as frozen core and frozen virtual orbitals, respectively. For the excitation operators in SAC and SAC-CI wave functions, all single and selected double excitations were included. A perturbation selection method ${ }^{59}$ was used. Threshold sets of "LevelTwo" were used, which is defined in Gaussian03 as $5.0 \times 10^{-6}$ and $5.0 \times$ $10^{-7}$ au for the SAC and SAC-CI wave functions, respectively.

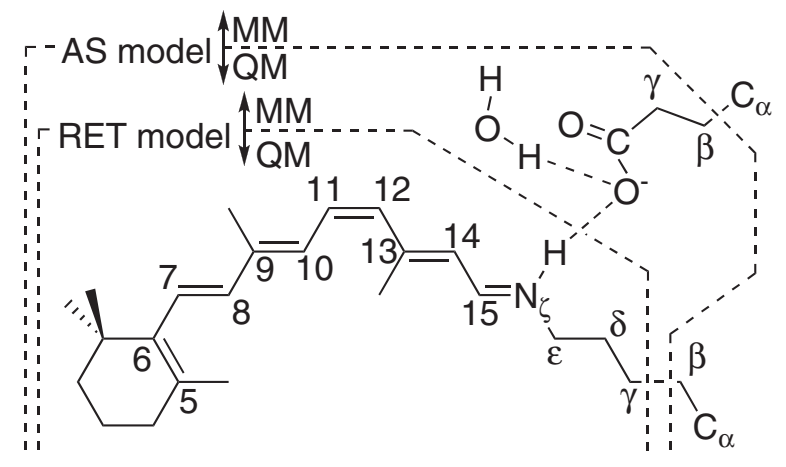

Figure 1. Schematic structure models of retinal PSB in the active site. The dotted lines show the QM/MM borders in the RET and AS models used for the QM/MM calculations. The $\mathrm{H}$ atoms are placed for terminating the QM boundary.
We also calculated excitation energy of retinal chromophore in gas phase and checked the basis set dependence using all-trans PSB model chromophore. To compare CASPT2 and SAC-CI results, we used atomic coordinates determined by a CASSCF calculation. ${ }^{31}$ The basis sets described above gave the excitation energy of $1.96 \mathrm{eV}$. We added the polarization functions on all atoms and got $1.98 \mathrm{eV}$. Diffuse $\mathrm{s}$ and $\mathrm{p}$ functions were further augmented, which gave $1.97 \mathrm{eV}$. The CASPT2 results $^{31}$ with the same CASCSF geometry were $2.27 \mathrm{eV}$. Present SAC-CI results were around $0.3 \mathrm{eV}$ smaller than the CASPT2 one. Since the CASSCF geometry used has a large bond-length alternation, the calculation might overestimate excitation energy. Even if we consider this possibility, the SAC-CI results do not so significantly deviate from the ELISA experiment $(2.03 \mathrm{eV}){ }^{60}$

We also examined the applicability of the present method by calculating excitation energy of 11-cis protonated Schiff base (11PSB) in hexane solution. ${ }^{61}$ The 11PSB and its counter ion, trichloroacetate, ${ }^{61}$ were described quantum-mechanically. Effect of solution was calculated with polarizable continuum model ( $\varepsilon=1.92$, heptane). Atomic coordinates were optimized using DFT at B3LYP/6-31G* level. Excited state calculation was performed using SAC-CI method with the same basis sets as we used for the cone pigments. For the $\mathrm{Cl}$ atoms, D95 basis set was employed. Excitation energy was calculated to be $2.59 \mathrm{eV}$ (at LevelTwo) and $2.61 \mathrm{eV}$ (at LevelThree), which reasonably agrees to the peak position observed in the experiment $\left(2.71 \mathrm{eV}^{61}\right)$. LevelThree energy threshold set defined in Gaussian03 is $1.0 \times$ $10^{-6}$ and $1.0 \times 10^{-7}$ au for SAC and SAC-CI wave functions, respectively.

Regarding the line shape of the spectra, we refer previous studies on the method ${ }^{21}$ and its application to the spectrum of retinal chromophore in protein. ${ }^{23}$

\section{Results and Discussion}

SAC-CI Theoretical Excitation Energies Compared with Experiments. The SAC-CI calculations showed reasonable agreement to the experimental excitation energies of six pigments, which are listed in Table 1. The SAC-CI calculations were performed with the AS model (Figure 1) under the electrostatic potential (ESP) of the MM region ("In opsin"), since this model gave reliable excitation energies of retinal proteins as investigated in our previous studies. ${ }^{17,45}$ The SACCI values for the wild-type $\mathrm{HB}, \mathrm{HG}$, and $\mathrm{HR}$ are 2.94, 2.32, and $2.08 \mathrm{eV}$, respectively, while the experimental absorption energies are $2.99,2.33$, and $2.20 \mathrm{eV}$, respectively. For the triple $(3 \mathrm{MUT})^{10}$ and double $(2 \mathrm{MUT})^{13}$ mutants of HG, the SAC-CI values were 2.20 and $2.40 \mathrm{eV}$, respectively, and the experimental values were 2.23 and $2.48 \mathrm{eV}$, respectively. The observed absorption peaks were assigned to the first excited states that have the largest oscillator strength in this energy region. They are $\pi-\pi^{*}$ HOMO-LUMO transitions. The present calculations do not include electronic polarization effect of proteins. In previous studies of bacteriorhodopsin, polarizable force field models gave a red shift of $0.19^{23}$ to $0.34 \mathrm{eV}^{62}$ A recent DFTB study gave smaller amount of red shift $(0.07 \mathrm{eV}) .{ }^{19,63}$

Physical Origin of Color Tuning. In the QM/MM calculations, we used two computational models, the "AS" and "RET" models as shown in Figure 1. In the AS model, the retinal PSB-lysine and the counter ion group were treated 
Table 1. Absorption Energies (in eV Unit) of HB, HG, HR, Rh, and Mutants

\begin{tabular}{|c|c|c|c|c|c|c|}
\hline \multirow{2}{*}{ Protein } & \multirow{2}{*}{\multicolumn{2}{|c|}{ Environment }} & \multirow{2}{*}{ QM size $\left.{ }^{a}\right)$} & \multicolumn{2}{|c|}{ Calcd } & \multirow{2}{*}{ Exptl/eV } \\
\hline & & & & $E_{\mathrm{ex}}{ }^{\mathrm{b})} / \mathrm{eV}$ & $f^{\mathrm{c})} / \mathrm{au}$ & \\
\hline \multirow[t]{4}{*}{ HB } & $\mathrm{WT}^{\mathrm{d})}$ & In opsin' ${ }^{\text {g) }}$ & AS & $2.94^{\mathrm{i})}$ & 1.16 & $2.99^{\mathrm{k})}$ \\
\hline & & & RET & 2.63 & 0.98 & - \\
\hline & & Bare $^{\text {h) }}$ & AS & 2.85 & 1.12 & - \\
\hline & & & RET & 1.61 & 0.62 & - \\
\hline \multirow[t]{6}{*}{ HG } & WT & In opsin & AS & $2.32^{\mathrm{i})}$ & 0.97 & $2.33^{1)}$ \\
\hline & & & RET & 1.99 & 0.82 & - \\
\hline & & Bare & AS & 2.48 & 1.04 & 一 \\
\hline & & & RET & 1.36 & 0.60 & - \\
\hline & $\left.3 \mathrm{MUT}^{\mathrm{e}}\right)$ & In opsin & AS & $2.20^{\mathrm{i})}$ & 0.91 & $2.23^{1)}$ \\
\hline & $\left.2 \mathrm{MUT}^{\mathrm{f}}\right)$ & In opsin & AS & $2.40^{\mathrm{i})}$ & 1.04 & $2.48^{\mathrm{m})}$ \\
\hline \multirow[t]{5}{*}{ HR } & WT & In opsin & AS & $2.08^{\mathrm{i})}$ & 0.95 & $2.20^{1)}$ \\
\hline & & & RET & 1.71 & 0.79 & - \\
\hline & & Bare & AS & 2.50 & 1.16 & - \\
\hline & & & RET & 1.33 & 0.67 & - \\
\hline & $\left.2 \mathrm{MUT}^{\mathrm{f}}\right)$ & Bare & AS & $2.28^{\mathrm{i})}$ & 1.01 & - \\
\hline \multirow[t]{4}{*}{$\mathrm{Rh}$} & WT & In opsin & AS & $2.45^{\mathrm{j})}$ & 1.03 & $2.49^{\mathrm{n})}$ \\
\hline & & & RET & $2.06^{\mathrm{j})}$ & 0.88 & - \\
\hline & & Bare & AS & 2.44 & 1.06 & - \\
\hline & & & RET & $1.36^{\mathrm{j})}$ & 0.63 & - \\
\hline
\end{tabular}

a) "AS" and "RET" denote AS and RET models. See Figure 1 for definition. b) Excitation energy.

c) Oscillator strength. d) Shows "Wild Type." e) Shows "Ala180Ser/Phe277Tyr/Ala285Thr" triple mutant.

f) Shows "His197Ala/Lys200Ala" double mutant. g) Including electrostatic potential (ESP) of MM region.

h) Without ESP of MM region. i) Ref. 39. j) Ref. 45. k) Ref. 9. 1) Ref. 10. m) Ref. 13. n) Ref. 6.

as a QM segment. The RET model was used to analyze the QM effect of the counter ion group. The calculated excitation energies in the opsin environment of the AS model, $E_{\mathrm{ex}}^{\mathrm{X}}(A S, O p \sin )$, were analyzed by

$$
E_{\mathrm{ex}}^{\mathrm{X}}(A S, \text { Opsin })=E_{\mathrm{ex}}^{\mathrm{X}}(R E T, \text { Bare })+E S^{\mathrm{X}}+Q M^{\mathrm{X}}
$$

where $\mathrm{X}=\mathrm{HB}, \mathrm{Rh}, \mathrm{HG}$, and HR. $E_{\mathrm{ex}}^{\mathrm{X}}($ RET, Bare $)$ denotes the excitation energy of the RET model without the protein environment (a "bare" chromophore model). $E S^{\mathrm{X}}$ is defined as $E_{\mathrm{ex}}^{\mathrm{X}}(R E T$, Opsin $)-E_{\mathrm{ex}}^{\mathrm{X}}(R E T$, Bare $)$ and accounts for the protein ES effect on the excitation energy. $E_{\mathrm{ex}}^{\mathrm{X}}(R E T, O p \sin )$ is the excitation energy of the RET model with the protein ES effect represented by a point charge model. $Q M^{\mathrm{X}}$ represents the counter ion residue $\mathrm{QM}$ effect and is evaluated by $E_{\mathrm{ex}}^{\mathrm{X}}(A S, O p \sin )-E_{\mathrm{ex}}^{\mathrm{X}}(R E T, O p \sin )$. The excitation energies of the additional two models, $E_{\mathrm{ex}}^{\mathrm{X}}(R E T, O p \sin )$ and $E_{\mathrm{ex}}^{\mathrm{X}}(R E T$, Bare $)$, are also given in Table 1. The geometries for calculating these excitation energies were the same as the $\mathrm{QM} / \mathrm{MM}$ optimized ones.

The $E S^{\mathrm{X}}$ term in eq 1 may be further decomposed as

$$
E S^{\mathrm{X}}=E S_{0}^{\mathrm{X}}+\delta E S^{\mathrm{X}}+R L X^{\mathrm{X}}
$$

The bare chromophore is first exposed to the protein ESP, which gives the first term.

$$
E S_{0}^{\mathrm{X}}=\sum_{\mathrm{A}}^{M M} \int \frac{\Delta \rho_{\mathrm{bare}}^{\mathrm{X}}(r) \cdot Q\left(r_{\mathrm{A}}\right)}{\left|r-r_{\mathrm{A}}\right|}
$$

Here $\Delta \rho_{\mathrm{bare}}^{\mathrm{X}}(r)$ is the electron density difference upon the transition of the bare chromophore, and $Q\left(r_{\mathrm{A}}\right)$ is the atomic charge of atom A. Next, the electron densities of the ground and excited states relax and give the relaxed density difference, $\Delta \rho^{\mathrm{X}}\left(=\Delta \rho_{\text {bare }}^{\mathrm{X}}+\Delta \gamma^{\mathrm{X}}\right)$, where $\Delta \gamma^{\mathrm{X}}$ represents the change due to the relaxation. This relaxation corrects the ES effect on the excitation energy as

$$
\delta E S^{\mathrm{X}}=\sum_{\mathrm{A}}^{M M} \int \frac{\Delta \gamma(r)^{\mathrm{X}} \cdot Q\left(r_{\mathrm{A}}\right)}{\left|r-r_{\mathrm{A}}\right|}
$$

The rest of the ES effect may arise from the electronic structure of the retinal PSB itself. They are represented as $R L X^{\mathrm{X}}$ $\left(=E S^{\mathrm{X}}-E S_{0}^{\mathrm{X}}-\delta E S^{\mathrm{X}}\right)$. The $\delta E S^{\mathrm{X}}$ and $R L X^{\mathrm{X}}$ terms represent the relaxation effect on the ES interaction and the chromophore's electronic structure, respectively, due to the protein ESP. Consequently, the excitation energy was decomposed into five terms.

To clarify the physical origin of the color tuning, each term was compared to that of Rh.

$$
\begin{aligned}
\Delta E_{\mathrm{ex}}^{\mathrm{X}}(A S, \text { Opsin })= & \Delta E_{\mathrm{ex}}^{\mathrm{X}}(R E T, \text { Bare })+\Delta E S_{0}^{\mathrm{X}} \\
& +\Delta \delta E S^{\mathrm{X}}+\Delta R L X^{\mathrm{X}}+\Delta Q M^{\mathrm{X}}
\end{aligned}
$$

Figure 2 clearly shows that the physical origin of color tuning is primarily the protein ES interaction between PSB and the opsin environment. In $\mathrm{HB}$, the overall ES effect $\left(\triangle E S^{\mathrm{X}}\right.$, sum of the second to 4-th terms in eq 5) amounts to $0.32 \mathrm{eV}$ of the overall spectral blue shift of $0.49 \mathrm{eV}$. The spectral red shifts of HG $(0.13 \mathrm{eV})$ and HR $(0.37 \mathrm{eV})$ are also dominated by the protein ES effect $(0.08 \mathrm{eV}$ for $\mathrm{HG}$ and $0.33 \mathrm{eV}$ for HR). The structural effect of the chromophore $(0.24 \mathrm{eV})$, as pointed out in a previous study, ${ }^{31}$ becomes important factor in the spectral 


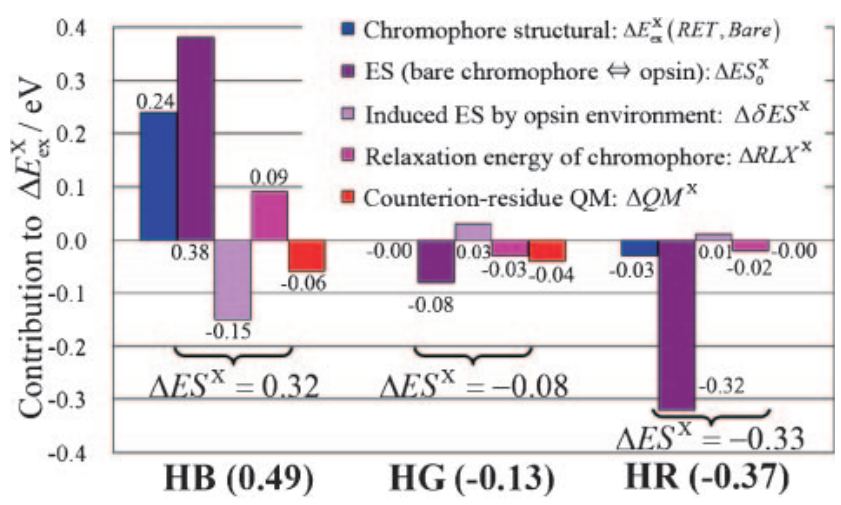

Figure 2. Physical origin of color tuning. Decomposition analysis on the absorption energy shifts. Rh was used as a reference. See eq 5 for the definitions of the terms. The data in the parenthesis shows total spectral shift compared to $\mathrm{Rh}$.

blue shift of HB. The counter ion QM effect gives essentially no contribution to the specific spectral shift among the cone pigments.

Within the ES terms, the $\Delta E S_{0}^{\mathrm{X}}$ term characterizes the ES effect on the color tuning, while the relaxation effects, $\Delta \delta E S^{\mathrm{X}}$ and $\Delta R L X^{\mathrm{X}}$ gave only minor corrections to the overall ES effect. This result indicates that the electronic structure in the gas phase characterizes qualitative feature of the spectral shift. A possibility of the electronic relaxation effect such as polarization of the retinal PSB can be ruled out.

There is another way to analyze the spectral tuning mechanism as conducted in previous studies. ${ }^{15,18,28}$ Starting with the calculation of the bare retinal chromophore, the counter ion was attached to the retinal as a part of the QM region, and then the rest of the protein environment was included as classical point charge models.

$$
E_{\mathrm{ex}}^{\mathrm{X}}(A S, O p \sin )=E_{\mathrm{ex}}^{\mathrm{X}}(R E T, \text { Bare })+\sigma_{\text {counter ion }}^{\mathrm{X}}+E S_{\text {other }}^{\mathrm{X}}
$$

The $\sigma_{\text {counter ion }}^{\mathrm{X}}$ term was defined as $E_{\mathrm{ex}}^{\mathrm{X}}(A S$, Bare $)-$ $E_{\mathrm{ex}}^{\mathrm{X}}(R E T$, Bare $)$ and represents the effect of the counter ion residue on the spectral tuning. The $E S_{\text {other }}^{\mathrm{X}}$ term was calculated as $E_{\mathrm{ex}}^{\mathrm{X}}(A S, O p \sin )-E_{\mathrm{ex}}^{\mathrm{X}}(A S$, Bare $)$ and includes the protein ES effect except for the counter ion. In the case of the spectral tuning of $\mathrm{Rh}$, since the $\sigma_{\text {counter ion }}^{\mathrm{X}}$ term gave a significant blue shift in previous CASPT2 studies $^{15,18,26,28,29}$ and others, ${ }^{16,30}$ the counter ion effect was ascribed to the origin of the spectral tuning in $\mathrm{Rh}^{15,18,28}$ On the $E S_{\text {other }}^{\mathrm{X}}$ term, Andruniów et al. ${ }^{28}$ and Coto et al. ${ }^{15}$ reported large red shift contribution of $0.45-0.53 \mathrm{eV}$, while minor red shift contribution $(0.08 \mathrm{eV})$ was obtained in recent study. ${ }^{18}$ In our present result, the excitation energy calculated with the AS model without the other ESP, $E_{\mathrm{ex}}^{\mathrm{X}}(A S$, Bare $)$, was $2.44 \mathrm{eV}$. Including the rest of the protein ESP only gave negligible change of $0.01 \mathrm{eV}$ in the $\mathrm{Rh}$ case, which is close to the conclusion given by Sekharan and co-workers. ${ }^{18}$

The each term in the eq 6 was compared to that of $\mathrm{Rh}$ as the same way as eq 5

$$
\begin{aligned}
\Delta E_{\mathrm{ex}}^{\mathrm{X}}(A S, \text { Opsin })= & \Delta E_{\mathrm{ex}}^{\mathrm{X}}(R E T, \text { Bare }) \\
& +\Delta \sigma_{\text {counter ion }}^{\mathrm{X}}+\Delta E S_{\text {other }}^{\mathrm{X}}
\end{aligned}
$$

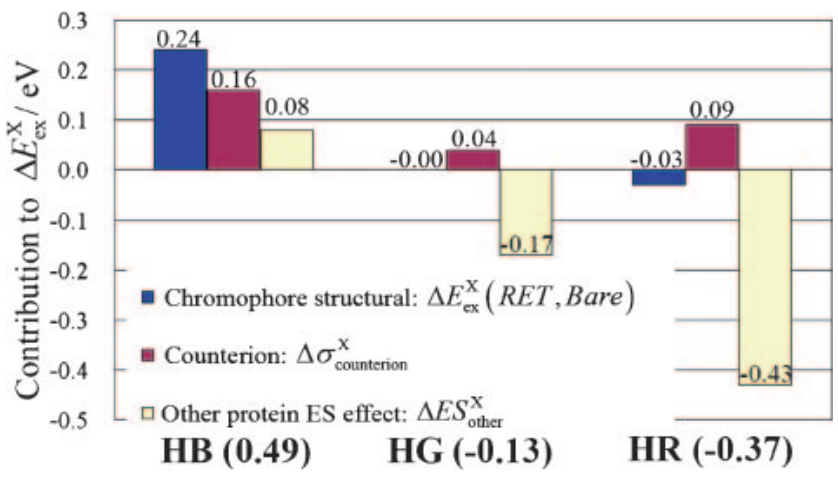

Figure 3. Decomposition analysis on the absorption energy shifts. See eq 7 for the definitions of the terms. The result of $\mathrm{Rh}$ was used as a reference. The data in the parenthesis shows total spectral shift compared to Rh.

where $X=H B, H G$, and HR. As clearly seen in Figure 3, the $\triangle E S_{\text {other }}^{\mathrm{X}}$ term is the determining factor of the color tuning among the cone pigments, which is consistent to the conclusion derived from eq 5 . The counter ion effect $\sigma_{\text {counter ion }}^{\mathrm{X}}$ plays a key role to determine the excitation energy of each pigment, but the relative order of the photoabsorption energy among the cone pigments is determined by the protein ESP other than the counter ion.

The protein ES effect on the excitation energy originates from the following mechanism. As shown in Figures 4a and $4 \mathrm{~b}$, the LUMO of the retinal chromophore is localized mainly on the Schiff base side (right) of the $\pi$-chain, while the HOMO is localized on the other $\beta$-ionone ring side (left). The HOMO-LUMO transition is, therefore, an intramolecular electron transfer from the ring to the $\mathrm{N}_{\zeta}$ region. As a result, the excitation is affected by the external ESP, which gradually decreases toward the $\mathrm{N}_{\zeta}$ atom in all the retinal proteins ${ }^{17,33,45}$ as shown in Figure $4 \mathrm{c}$. The $\mathrm{N}_{\zeta}$-to- $\mathrm{C}_{5}$ differences in the ESP are $-0.15,-0.11,-0.10$, and -0.07 au for $\mathrm{HB}, \mathrm{Rh}, \mathrm{HG}$, and $\mathrm{HR}$, respectively. Under these protein ESPs, the HOMO-LUMO gaps increase by $0.69,0.44,0.37$, and $0.15 \mathrm{eV}$ in $\mathrm{HB}, \mathrm{Rh}$, $\mathrm{HG}$, and HR, respectively, in comparison with the isolated "bare" chromophore. Table 1 clearly shows that the excitation energies of the pigments correlate well with the $\mathrm{N}_{\zeta}$-to- $\mathrm{C}_{5}$ ESP differences, which were summarized in "ES" term in Figure 2 and "Other protein ES effect" in Figure 3.

The structural effect of the chromophore in HB originates from the bond length alternation (BLA) of the retinal $\pi$ skeleton. As shown in Figure 5, the BLA enlarges in the order of $\mathrm{HB}>\mathrm{HG}>\mathrm{HR}$. When BLA is prominent, the absorption energy of the first excited state becomes large, as shown both experimentally ${ }^{64}$ and theoretically. ${ }^{30,45}$ The order of BLA among the cone pigments explains the results of the resonance Raman experiment: the vibrational frequencies of the $\mathrm{C}=\mathrm{C}$ ethylenic stretching and the $\mathrm{C}=\mathrm{NH}$ stretching modes of $\mathrm{HB}$ are the largest among the cone pigments. ${ }^{65}$

When BLA is larger, the rotation around the $\mathrm{C}-\mathrm{C}$ single bond in the $\pi$-chain becomes easier. The distortion around the $\mathrm{C}_{6}-\mathrm{C}_{7}$ single bond (rotation of $\beta$-ionone ring) in $\mathrm{HB}$ is 56 degrees from the molecular plane, about 10-18 degrees larger than those of the other pigments. The $\mathrm{C}_{6}-\mathrm{C}_{7}$ rotation remarkably increases the excitation energy of the PSB. ${ }^{31,45}$ 
(a)LUMO

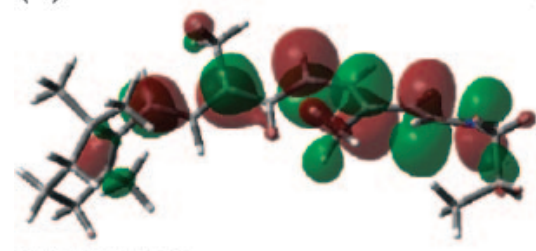

(b)HOMO

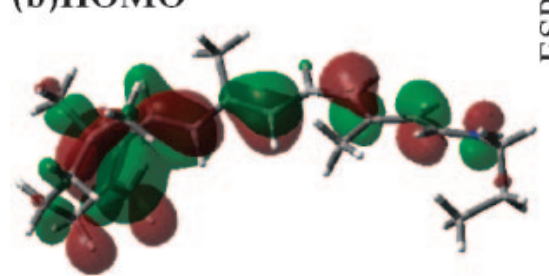

(c)Protein-electrostatic potential

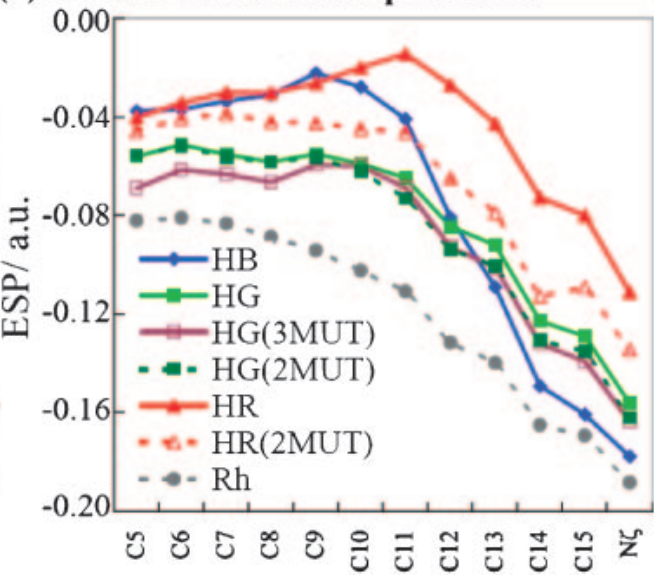

Figure 4. MO distributions and protein-ESP. (a) LUMO and (b) HOMO distributions of the retinal PSB. (c) Protein ESP at each atom of the retinal $\pi$ skeleton in the RET model.

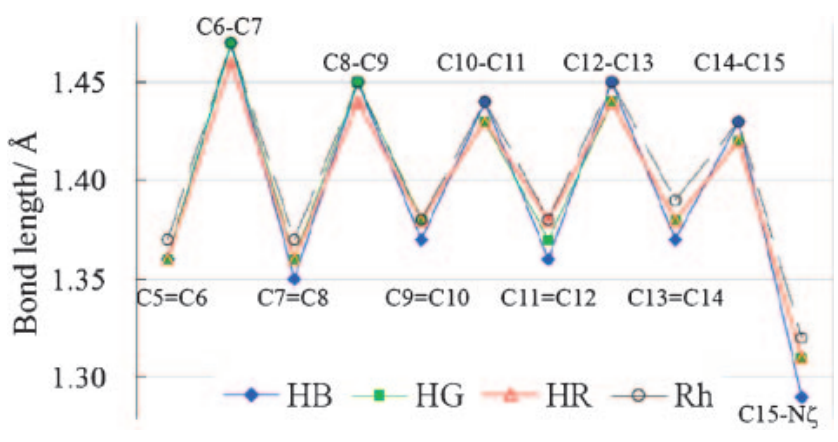

Figure 5. Bond lengths/Å between conjugated atoms in the retinal $\pi$-skeleton.

The $\mathrm{C}_{8}-\mathrm{C}_{9}$ and $\mathrm{C}_{10}-\mathrm{C}_{11}$ single bonds of $\mathrm{HB}$ also deviate by $6-13$ and $13-15$ degrees, respectively (See the list of dihedral angles in $\mathrm{SI}$ ).

The BLA is actually caused by the protein ESP. As the ESP difference between the $\mathrm{N}_{\zeta^{-}}$and $\mathrm{C}_{5}$-edges increases, the BLA becomes more significant (See SI). Thus, the protein environment tunes the excitation energy of PSB by controlling the BLA of the $\pi$-skeleton.

Amino Acid Sequences Responsible for Color Tuning. The protein ES effect is expressed further as a sum of the contribution from each amino acid $I$ as

$$
\begin{array}{r}
E S^{\mathrm{X}}(I)=\sum_{\mathrm{A} \in I} \int \frac{\Delta \rho(r) \cdot Q\left(r_{\mathrm{A}}\right)}{\left|r-r_{\mathrm{A}}\right|} \mathrm{d} r \\
(\mathrm{X}=\mathrm{HB}, \mathrm{Rh}, \mathrm{HG}, \text { and HR })
\end{array}
$$

where $\Delta \rho(r)$ is the electron density difference upon transition and $Q\left(r_{\mathrm{A}}\right)$ is the atomic charge of atom A of the amino acid $I$. To search for important positions of $I$, the relative numbers $\Delta E S^{\mathrm{X}-\mathrm{Y}}(I)=E S^{\mathrm{X}}(I)-E S^{\mathrm{Y}}(I)$ were calculated for all $I$ and for all pairs of pigments $\mathrm{X}$ and $\mathrm{Y}(\mathrm{X}, \mathrm{Y}=\mathrm{HB}, \mathrm{Rh}, \mathrm{HG}$, and HR). Charged residues and their counter ions were treated as single residues. The important amino acids are described in Figure 6.

These amino acid positions may be classified into four groups, depending on their specific roles. The first group of amino acids works to discriminate $\mathrm{HB}$ from $\mathrm{Rh}, \mathrm{HG}$, and $\mathrm{HR}$.
The largest contribution comes from position 186 . It is very interesting that the common amino acid, serine, gives red shift contributions of $0.09-0.13 \mathrm{eV}$ in $\mathrm{Rh}, \mathrm{HG}$, and $\mathrm{HR}$, while it is very small in HB. We note that this fact cannot be extracted only from the one-dimensional amino acid sequence information: the structural and physical knowledge of the molecular interactions is important. The structures of the retinal binding sites are shown in Figure 7. In Ser186 of Rh and in Ser202 of $\mathrm{HG}$ and $\mathrm{HR}$, the $\mathrm{OH}$ directions are parallel to the retinal $\pi$-skeleton, resulting in a spectral red shift, since they create a positive ESP around the Schiff base part. The $\mathrm{OH}$ group in $\mathrm{HB}$, however, is perpendicular to the $\pi$-skeleton, so its effect is negligible. These $\mathrm{OH}$ directions are controlled by the hydrogen-bonding networks and are energetically stable. Although we artificially tried to change the bonding pattern in HB into that of $\mathrm{Rh}$, it returned to the original $\mathrm{HB}$ pattern after the QM/ $\mathrm{MM}$ energy minimization. We found that this machinery for $\mathrm{HB}$ and $\mathrm{Rh}^{17}$ is actually common to all the visual pigments.

In a previous experiment, an Ala308Ser mutation experiment (at position 292 in Figure 6) was performed in a human red His197Tyr mutant, and a spectral blue shift of $0.08 \mathrm{eV}$ was observed. ${ }^{67}$ In addition, a Ser308Ala mutant was produced in the mouse green cone pigment, which showed a red shift of $0.08 \mathrm{eV}^{67,68}$ These results indicate that a serine residue at position 292 increases the excitation energy. These experimental results seem to arise from the following two reasons. First, Ala308 in HR itself has the red shift effect as shown in Figure 6. Second, as seen in Figure 7a, a serine at position 292 mediates the hydrogen-bonding network. This residue tunes the excitation energy by controlling the $\mathrm{OH}$ direction of serine residues at position 186 . If an alanine is at position 292, a serine at 186 would contribute to the spectral red shift as in $\mathrm{Rh}$, $\mathrm{HG}$, and HR.

A previous mutation study by Lin and co-workers demonstrated that Gly90Ser, Trp265Tyr, and Ala292Ser mutations in $\mathrm{Rh}$ show particularly large spectral blue shifts. ${ }^{12}$ Fasick and co-workers showed, however, that the opposite mutations in HB did not necessarily give spectral red shifts, ${ }^{9}$ as shown in Table 2. The results of the decomposition analysis, $\Delta E S^{\mathrm{X}-\mathrm{Y}}(I)=E S^{\mathrm{X}}(I)-E S^{\mathrm{Y}}(I) \quad(\mathrm{X}=\mathrm{HB}, \quad \mathrm{Y}=\mathrm{Rh}), \quad$ were 


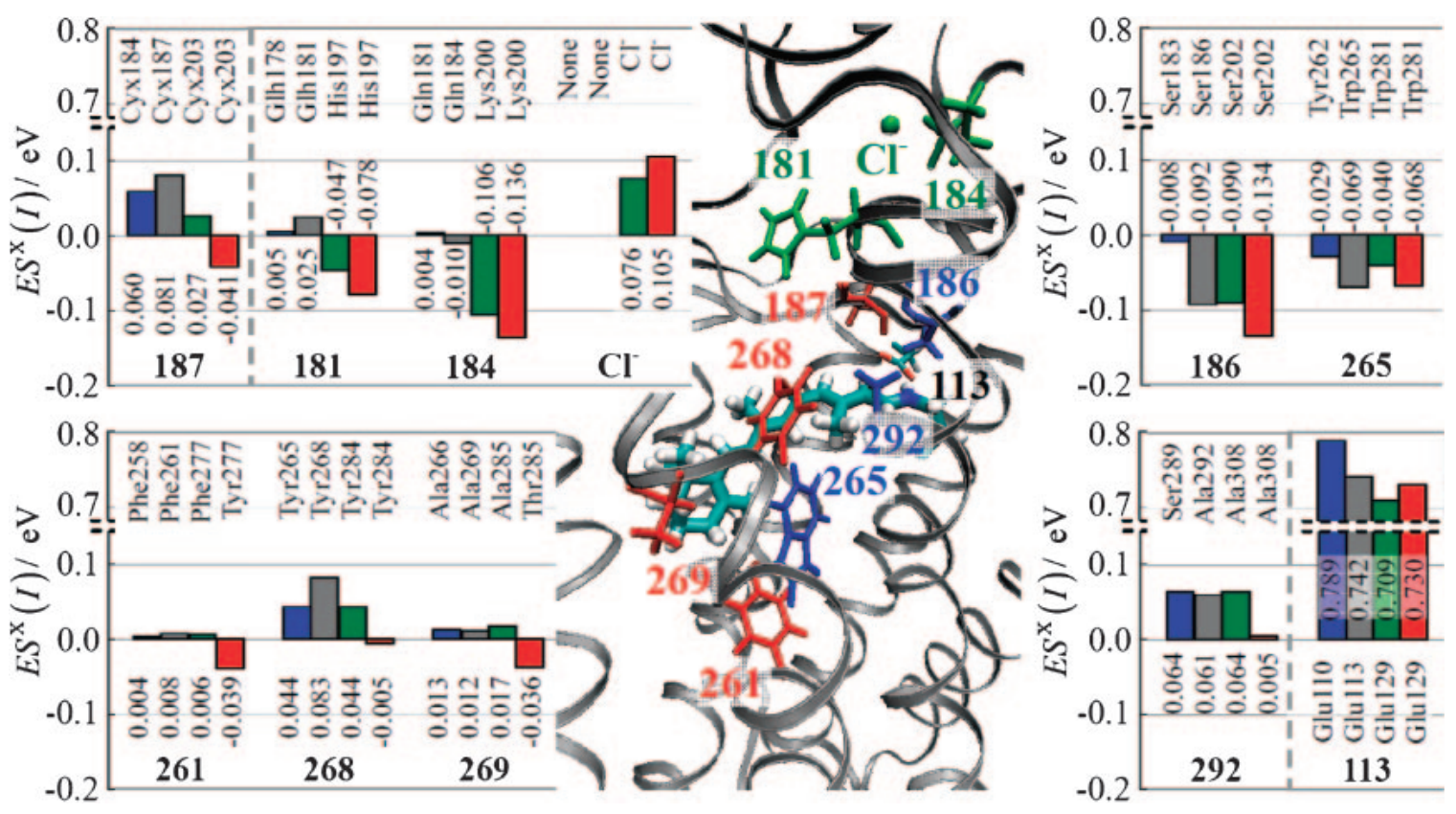

Figure 6. Amino acid sequences important for color tuning. The names of the residues and their ES contributions to the excitation energies $\left(E S^{\mathrm{X}}(I)\right.$ in $\left.\mathrm{eV}\right)$ are given for HB (blue), Rh (gray), HG (green), and HR (red).

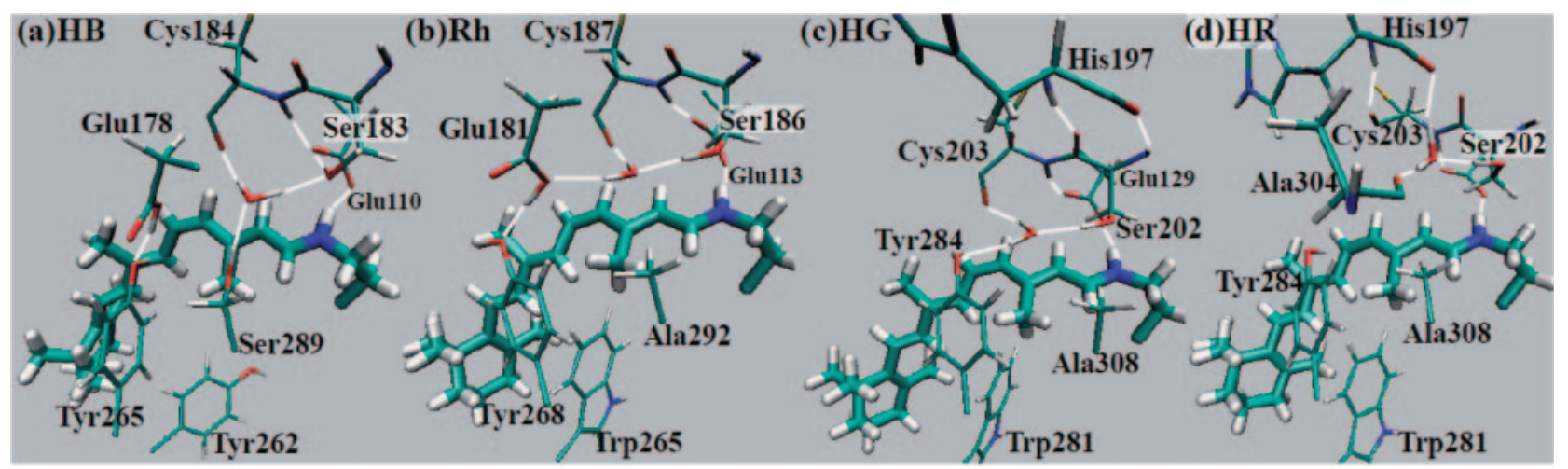

Figure 7. Some amino acids, which are important for color tuning. Hydrogen bonding is indicated by a white line. (a) HB, (b) Rh, (c) HG, and (d) HR visual cone pigments. Structures were obtained by the QM/MM optimization. VMD ${ }^{66}$ was used for the graphics.

compared with those of the two experimental studies in Table 2. The theoretical results were closer to those of Fasick and co-workers. ${ }^{9}$ Ser87 in HB (Gly90 in Rh) contributes to the red shift $(-0.018 \mathrm{eV})$, while Tyr262 and Ser289 in HB (Trp265 and Ala292 in Rh, respectively) contribute to blue shifts of 0.040 and $0.004 \mathrm{eV}$, respectively. This would imply that the mutations in $\mathrm{Rh}^{12}$ cause structural changes that induce other sources of the spectral shift.

Table 2 also shows 5 additional amino acids that gave large ES contributions to the spectral shift. Although amino acid residues in positions 113,118 , and 186 are identical between $\mathrm{HB}$ and $\mathrm{Rh}$, their red shift contributions were estimated to be $0.049,0.030$, and $0.084 \mathrm{eV}$, respectively. These results clearly show that one-dimensional amino acid sequence information alone is not sufficient to understand the color tuning mechanism of the cone pigments. Three-dimensional structural information and the physical interactions involved are essential to understand the mechanism.
The second group is the $\mathrm{Cl}^{-}$binding site, which is composed of positions 181, 184, and the $\mathrm{Cl}^{-}$ion. As shown in Figure 6, the overall contribution of the $\mathrm{Cl}^{-}$binding site is the spectral red shift in both $\mathrm{HG}$ and $\mathrm{HR}$, although the $\mathrm{Cl}^{-}$itself has a blue shift contribution. Positions 181 and 184 in HG and HR are positively charged histidine and lysine, respectively, which have red shift contributions of $0.05-0.14 \mathrm{eV}$. In contrast, $\mathrm{HB}$ and $\mathrm{Rh}$ have neutral glutamate and glutamine residues at positions 181 and 184 , respectively, which give almost no contributions.

Structures around the $\mathrm{Cl}^{-}$binding sites in $\mathrm{HG}$ and $\mathrm{HR}$ are shown in Figure 8. The conformations of His197 in HG and HR are different. This arises from the differences in the local cavity environment and hydrogen-bonding pattern. As shown in Figures 7c and 7d, the main chain part of His 197 interacts with Ser202 in HG but with Cys203 in HR. Consequently, the $\mathrm{C}=\mathrm{O}$ orientation in Cys 203 changes in $\mathrm{HR}$, which results in the spectral red shift. It is, however, very difficult to determine the 
Table 2. Comparison of Theoretical Spectral Shifts from Rh to $\mathrm{HB}$ with the Experimental Results (in eV Unit)

\begin{tabular}{ccccc}
\hline \multirow{2}{*}{ HB } & \multirow{2}{*}{ Rh } & \multirow{2}{*}{$\Delta E S^{\text {HB-Rh }}(I)^{\mathrm{a})}$} & \multicolumn{2}{c}{ Exptl } \\
\cline { 4 - 5 } & & & Rh mut. $^{\mathrm{b})}$ & HB mut. ${ }^{\mathrm{c}}$ \\
\hline Leu83 & Met86 & 0.016 & - & - \\
Ser87 & Gly90 & -0.018 & 0.056 & -0.049 \\
Gly114 & Ala117 & -0.032 & 0.020 & - \\
Leu119 & Glu122 & 0.001 & 0.025 & - \\
Thr121 & Ala124 & -0.013 & 0.015 & - \\
Tyr262 & Trp265 & 0.040 & 0.077 & 0.067 \\
Ser289 & Ala292 & 0.004 & 0.045 & 0.000 \\
Ser292 & Ala295 & -0.041 & 0.025 & - \\
Cys296 & Ala299 & 0.011 & 0.010 & - \\
(9 contributions) & $-0.032^{\mathrm{d})}$ & $0.357^{\mathrm{e})}$ & - \\
& & & & \\
Phe40 & Tyr43 & 0.032 & - & - \\
Glu110 & Glu113 & 0.049 & - & - \\
Thr115 & Thr118 & 0.030 & - & - \\
Ser183 & Ser186 & 0.084 & - & - \\
Leu204 & Met207 & 0.030 & - & - \\
(5 contributions) & $0.225^{\mathrm{f})}$ & - & - \\
\hline
\end{tabular}

a) Defined by $\Delta E S^{\mathrm{HB}-\mathrm{Rh}}(I)=E S^{\mathrm{HB}}(I)-E S^{\mathrm{Rh}}(I)$. b) $\mathrm{Rh}$ mutant. $^{12}$ c) HB mutant. ${ }^{9}$ d) Sum of the 9 residues' contributions. e) Spectral shift observed in the 9-ple mutant of Rh, (Met86Leu, Gly90Ser, Ala117Gly, Glu122Leu, Ala124Thr, Trp265Tyr, Ala292Ser, Ala295Ser, and Ala299Cys). f) Sum of the 5 residues' contributions.

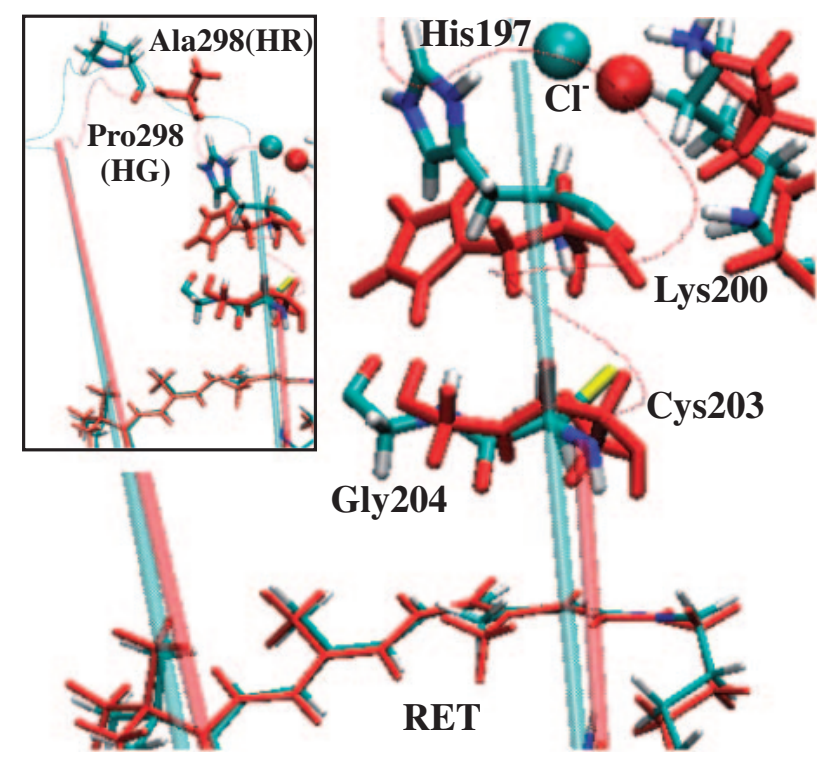

Figure 8. Structure of the $\mathrm{Cl}^{-}$binding site in $\mathrm{HG}$ (colored by elements) and HR (red) obtained by the QM/MM calculations. The inset shows amino acids at positions 298 .

origin of this structural difference. Our tentative opinion, based on the observation of the structure, is as follows: In the cases of bacteriorhodopsin (bR) and sensoryrhodopsin II (sR II), a small perturbation in the loop structure causes the spectral shift. ${ }^{69}$ As shown in Figure 8, the loop between transmembrane helices 6 and 7 in HG becomes shorter than that in HR due to the Pro298 residue (Ala298 in HR). This difference slightly changes the
(a)HG

(b)HR ESP by retinal/a.u.

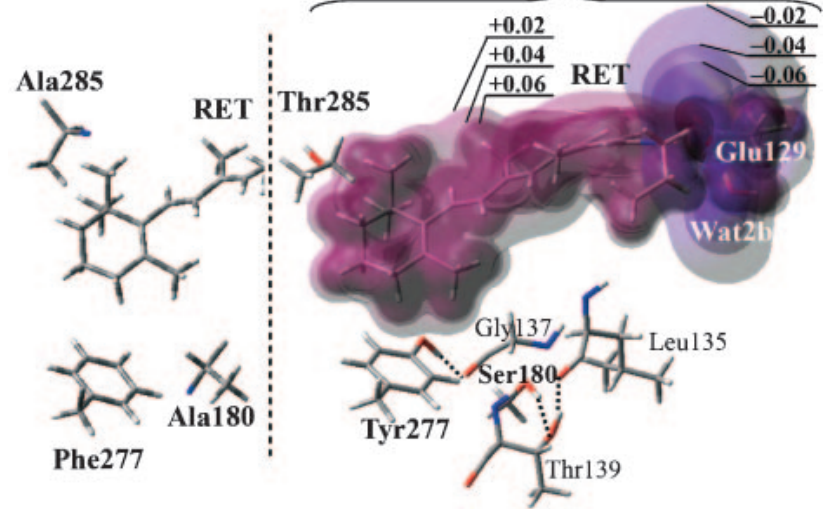

Figure 9. Three amino acids in (a) HG and (b) HR active sites. The ESP created by the retinal PSB is also shown. Positive and negative areas are indicated by deep red and purple, respectively.

interactions between amino acids, which might ultimately result in a different orientation of the His197 residue.

The red shift effect of the $\mathrm{Cl}^{-}$binding site was confirmed by theoretical mutation simulations. As in the previous experimental mutagenesis, ${ }^{13}$ His 197 and Lys200 in HG and HR were replaced by alanine residues to remove the $\mathrm{Cl}^{-}$binding site. The calculated excitation energy of the HG mutant became $2.40 \mathrm{eV}$, which was in close accordance with the observed value, $2.48 \mathrm{eV}$. For the HR double mutant, the spectral blue shift of $0.20 \mathrm{eV}$ was expected as shown in Table 1. The ESP curves shown in Figure 4c also explain the spectral blue shifts caused by the mutations. The ESPs of 2MUTs of HG and HR decrease around the SB regions, when the $\mathrm{Cl}^{-}$binding sites were removed.

The third group (positions 187, 261, 268, and 269) characterizes HR. At positions 261 and 269, HR has dipolar Tyr277 and Thr285, whereas HB, HG, and Rh have nonpolar phenylalanine and alanine residues, ${ }^{10}$ as shown in Figure 9. The $\mathrm{OH}$ groups in $\mathrm{HR}$ align such that the $\mathrm{O}$ atoms are closer to the retinal chromophore since the ESP created by the retinal PSB is positive around the $\beta$-ionone side (Figure $9 \mathrm{~b}$ ). These $\mathrm{OH}$ orientations provide a negative ESP on the $\beta$-ionone side of the chromophore, which decreases the absorption energy. Although all pigments have a cysteine residue at position 187 , only Cys203 in HR has a red shift effect, as shown in Figure 6. This difference arises from the $\mathrm{C}=\mathrm{O}$ orientation of the peptide bond which is controlled by the local hydrogen-bonding network, as depicted in Figure 7.

These $\mathrm{OH}$ dipole contributions were also confirmed by theoretical mutation. The neutral residues Ala180, Phe277, and Ala285 in HG were replaced by the dipolar residues in HR, Ser, Tyr, and Thr, respectively. The calculated excitation energy of the triple mutant was $2.20 \mathrm{eV}$, which is very close to the experimentally created triple mutant $(2.23 \mathrm{eV})$. As seen in Figure $4 c$, the dipolar residues introduced in HG (3MUT) provide negative ESP contributions around the $\beta$-ionone side of the chromophore, which decrease the absorption energy.

We further compared the ES contributions of the amino acids with the results of the experimental mutagenesis. ${ }^{10}$ Around 
Table 3. Spectral Shift by the 7 Residues (in eV Unit) ${ }^{\text {a) }}$

\begin{tabular}{ccccc}
\hline \multirow{2}{*}{ HG } & \multirow{2}{*}{ HR } & \multirow{2}{*}{$\Delta E S^{\mathrm{HG}-\mathrm{HR}}(I)^{\mathrm{b})}$} & \multicolumn{2}{c}{ Exptl $^{\mathrm{c})}$} \\
\cline { 4 - 5 } & & & HG mut. & HR mut. \\
\hline Tyr116 & Ser116 & 0.004 & - & - \\
Ala180 & Ser180 & 0.013 & 0.009 & 0.028 \\
Thr230 & Ile230 & 0.001 & 0.000 & 0.008 \\
Ser233 & Ala233 & 0.002 & 0.004 & 0.012 \\
Phe277 & Tyr277 & 0.044 & 0.026 & 0.040 \\
Ala285 & Thr285 & 0.053 & 0.043 & 0.064 \\
Phe309 & Tyr309 & 0.013 & 0.000 & 0.008 \\
& & & & \\
3 contributions & $0.111^{\mathrm{f})}$ & $0.097^{\mathrm{g})}$ & - \\
7 contributions & $0.131^{\mathrm{h})}$ & $0.128^{\mathrm{i})}$ & - \\
\hline
\end{tabular}

a) $\mathrm{HR}$ is taken as reference. b) Defined by $\Delta E S^{\mathrm{HG}-\mathrm{HR}}(I)=$ $E S^{\mathrm{HG}}(I)-E S^{\mathrm{HR}}(I)$. c) Ref. 10. d) Mutation of HG. e) Mutation of HR. f) Sum of the 3 residues at the positions 180, 277, and 285. g) Ala180Ser, Phe277Tyr, and Ala285Thr triple mutant of HG. h) Sum of the 7 residues. i) Phe116Ser, Ala180Ser, Thy230Ile, Ser233Ala, Phe277Tyr, Ala285Thr, and Phe309Tyr septuple mutant of HG.

$80 \%$ of the spectral shift between HG and HR was ascribed to the three amino acids at positions 180,277 , and $285 .{ }^{10}$ The majority of the spectral difference was explained by up to seven amino acids. ${ }^{10}$ We compared the experimental spectral shifts ${ }^{10}$ with the results of the decomposition analysis given by $\Delta E S^{\mathrm{X}-\mathrm{Y}}(I)=E S^{\mathrm{X}}(I)-E S^{\mathrm{Y}}(I) \quad(\mathrm{X}=\mathrm{HG}, \mathrm{Y}=\mathrm{HR}) . \quad$ These results are summarized in Table 3 . In the present analysis, these residues contribute to the red-to-green spectral shift by $0.001-$ $0.053 \mathrm{eV}$, and the total blue shift amounts to $0.131 \mathrm{eV}$. Particularly, the three residues at positions 180, 277, and 285 give large contributions and amount to $0.111 \mathrm{eV}$. Our theoretical results closely reproduce the experimental spectral shifts (0.128 and $0.097 \mathrm{eV}$ for the septuple and triple mutants, respectively), which support our protein structures and electronic structures described in the present study.

The last group (position 113) is the counter ion residue of the PSB. The amount of the red shifts is in the order of $\mathrm{HB}>\mathrm{Rh}>\mathrm{HR}>\mathrm{HG}$, indicating that the counter ion residues are qualitatively controlling the spectral peak positions. According to the results with the AS model shown in Figure 3, the counter ion effect does not explain the relative order of the excitation energies among the visual pigments.

Thus, we found that color tuning is regulated by the amino acids at specific positions in the amino acid sequence of the retinal proteins. This fact may imply that there are some genetic origins (for a review, see reference ${ }^{4}$ ). The present theoretical study has thus shed light on the physical and chemical origins of color tuning behind the existing experimental findings.

\section{Conclusion}

Human retina has $\mathrm{HR}, \mathrm{HG}$, and $\mathrm{HB}$ cone pigments which are responsible for the color vision. Photoabsorption wavelengths of the cone pigments spread uniquely over the three primary colors. These pigments, however, include only one common chromophore, retinal, which means the protein environment, opsin, regulates excitation energy of retinal chromophore. In this article, we report physical basis of the color tuning in human vision on the basis of SAC-CI calculations for excited states of the cone pigments.

The present SAC-CI results showed reasonable agreements with experimental photoabsorption energies of three cone pigments and their mutants. Decomposition analysis which clarifies the individual role of the amino acids also showed good agreements with the results of mutation experiments. Computational models of the cone pigments were obtained theoretically by QM/MM geometry optimization. Starting with the homology models provided in a previous study, we modified protonation states of ionizable residues and position of water molecules using Poisson-Boltzmann calculations and available experimental data. QM(B3LYP)/MM(AMBER) calculations were performed for geometry optimizations.

Two kinds of analysis were conducted for clarifying physical origin of the color tuning. Both showed that the dominant origin of the red-green-blue distinction lies in differences in electrostatic interactions between retinal and its surrounding proteins. Structural distortion effect of the retinal chromophore is important in human blue pigment. Counter ion residue contributes significant blue shift effect ${ }^{15,18,28}$ and, therefore, important for determining the excitation energy of each cone pigment. However, according to the present analysis, the counter ion is not relevant to the relative order of the excitation energies among the visual pigments.

Detailed analysis on the individual roles of the amino acids within these proteins has clarified elaborate mechanisms of the color tuning, in accordance with previous mutagenesis $9,10,13$ experiments. Previous mutation experiments clarified the key amino acids for the color tuning on the basis of the homology/ difference among the amino acid sequences. Present study adds three-dimensional understanding on structures, electronic wave functions, and molecular interactions. Furthermore, the color tuning is regulated by amino acids at specific positions in the opsins, suggesting some genetic origins for the color tuning.

This study was supported by a Grant-in-Aid for Young Scientists, Scientific Research on Priority Areas "Molecular theory for real systems," and the Global COE Program (No. B-09) from JSPS. A part of the study was also supported by JST-CREST and Grant-in-Aid for Young Scientists from ACCMS and IIMC, Kyoto University. A portion of the computations was carried out at RCCS (Okazaki, Japan).

\section{Supporting Information}

Descriptions of methodology and computations, complete ref., 52 the QM/MM optimized structures of retinal chromophores, the effect of the external ESP on the BLA, the SAC-CI results with the RET models, and Gaussian inputs for the SAC-CI calculations. These materials are available free of charge on the Web at: http:// www.csj.jp/journals/bcsj/.

\section{References}

1 G. Wald, Nature 1968, 219, 800.

2 S. L. Merbs, J. Nathans, Nature 1992, 356, 433.

3 D. D. Oprian, A. B. Asenjo, N. Lee, S. L. Pelletier, Biochemistry 1991, 30, 11367.

4 Y. Shichida, H. Imai, Cell. Mol. Life Sci. 1998, 54, 1299.

5 R. A. Mathies, J. Lugtenburg, in Handbook of Biological 
Physics, ed. by D. G. Stavenga, W. J. DeGrip, E. N. Pugh, Elsevier Science B. V., Amsterdam, 2000, Vol. 3, p. 55.

6 H. Kandori, Y. Shichida, T. Yoshizawa, Biochemistry (Moscow, Russ. Fed.) 2001, 66, 1197.

7 R. M. Boynton, Human Color Vision. Holt, Rinehart and Winston, New York, 1979.

8 H. J. A. Dartnall, J. K. Bowmaker, J. D. Mollon, Proc. R. Soc. London, Ser. B. 1983, 220, 115.

9 J. I. Fasick, N. Lee, D. D. Oprian, Biochemistry 1999, 38 , 11593.

10 A. B. Asenjo, J. Rim, D. D. Oprian, Neuron 1994, 12, 1131.

11 J. Nathans, D. Thomas, D. S. Hogness, Science 1986, 232, 193.

12 S. W. Lin, G. G. Kochendoerfer, K. S. Carroll, D. Wang, R. Mathies, T. P. Sakmar, J. Biol. Chem. 1998, 273, 24583.

13 Z. Wang, A. B. Asenjo, D. D. Oprian, Biochemistry 1993, 32,2125 .

14 M. Neitz, J. Neitz, G. H. Jacobs, Science 1991, 252, 971.

15 P. B. Coto, A. Strambi, N. Ferré, M. Olivucci, Proc. Natl. Acad. Sci. U.S.A. 2006, 103, 17154.

16 M. Hoffmann, M. Wanko, P. Strodel, P. H. König, T. Frauenheim, K. Schulten, W. Thiel, E. Tajkhorshid, M. Elstner, J. Am. Chem. Soc. 2006, 128, 10808.

17 K. Fujimoto, J. Hasegawa, S. Hayashi, H. Nakatsuji, Chem. Phys. Lett. 2006, 432, 252.

18 S. Sekharan, M. Sugihara, V. Buss, Angew. Chem., Int. Ed. 2007, 46, 269.

19 M. Wanko, M. Hoffmann, T. Frauenheim, M. Elstner, J. Phys. Chem. B 2008, 112, 11462.

20 A. Warshel, J. Phys. Chem. 1979, 83, 1640.

21 V. Luzhkov, A. Warshel, J. Am. Chem. Soc. 1991, 113, 4491.

22 A. Warshel, Z. T. Chu, J.-K. Hwang, Chem. Phys. 1991, $158,303$.

23 A. Warshel, Z. T. Chu, J. Phys. Chem. B 2001, 105, 9857.

24 S. Hayashi, E. Tajkhorshid, E. Pebay-Peyroula, A. Royant, E. M. Landau, J. Navarro, K. Schulten, J. Phys. Chem. B 2001, 105, 10124.

25 T. Vreven, K. Morokuma, Theor. Chem. Acc. 2003, 109, 125 .

26 M. Schreiber, V. Buß, M. Sugihara, J. Chem. Phys. 2003, $119,12045$.

27 N. Ferré, M. Olivucci, J. Am. Chem. Soc. 2003, 125, 6868.

28 T. Andruniów, N. Ferré, M. Olivucci, Proc. Natl. Acad. Sci. U.S.A. 2004, 101, 17908.

29 J. Hufen, M. Sugihara, V. Buss, J. Phys. Chem. B 2004, 108, 20419.

30 M. Wanko, M. Hoffmann, P. Strodel, A. Koslowski, W. Thiel, F. Neese, T. Frauenheim, M. Elstner, J. Phys. Chem. B 2005, 109, 3606 .

31 A. Cembran, R. González-Luque, P. Altoè, M. Merchán, F. Bernardi, M. Olivucci, M. Garavelli, J. Phys. Chem. A 2005, 109, 6597.

32 R. J. Trabanino, N. Vaidehi, W. A. Goddard, III, J. Phys. Chem. B 2006, 110, 17230.

33 K. Fujimoto, J. Hasegawa, S. Hayashi, S. Kato, H. Nakatsuji, Chem. Phys. Lett. 2005, 414, 239.

34 K. Bravaya, A. Bochenkova, A. Granovsky, A. Nemukhin, J. Am. Chem. Soc. 2007, 129, 13035.

35 H. Nakatsuji, K. Hirao, J. Chem. Phys. 1978, 68, 2053.

36 H. Nakatsuji, Chem. Phys. Lett. 1978, 59, 362.

37 H. Nakatsuji, Chem. Phys. Lett. 1979, 67, 329.
38 H. Nakatsuji, Chem. Phys. Lett. 1979, 67, 334.

39 K. Fujimoto, J. Hasegawa, H. Nakatsuji, Chem. Phys. Lett. 2008, 462, 318.

40 M. Ehara, J. Hasegawa, H. Nakatsuji, in Theory and Applications of Computational Chemistry, ed. by C. Dykstra, G. Frenking, K. Kim, G. Scuseria, Elsevier Science, New York, 2006.

41 J. Hasegawa, H. Nakatsuji, in Radiation Induced Molecular Phenomena in Nucleic Acid: A Comprehensive Theoretical and Experimental Analysis, ed. by M. Shukla, J. Leszczynsky, Springer, 2008, p. 93.

42 H. Nakatsuji, J. Hasegawa, K. Ohkawa, Chem. Phys. Lett. 1998, 296, 499.

43 J. Hasegawa, K. Ohkawa, H. Nakatsuji, J. Phys. Chem. B 1998, 102, 10410.

44 J. Hasegawa, H. Nakatsuji, Chem. Lett. 2005, 34, 1242.

45 K. Fujimoto, S. Hayashi, J. Hasegawa, H. Nakatsuji, J. Chem. Theory Comput. 2007, 3, 605.

46 R. E. Stenkamp, S. Filipek, C. A. G. G. Driessen, D. C. Teller, K. Palczewski, Biochim. Biophys. Acta 2002, 1565, 168.

47 D. Bashford, K. Gerwert, J. Mol. Biol. 1992, 224, 473.

48 T. Okada, Y. Fujiyoshi, M. Silow, J. Navarro, E. M. Landau, Y. Shichida, Proc. Natl. Acad. Sci. U.S.A. 2002, 99, 5982.

49 A. Warshel, M. Levitt, J. Mol. Biol. 1976, 103, 227.

50 S. Hayashi, I. Ohmine, J. Phys. Chem. B 2000, 104, 10678.

51 J. Hasegawa, K. Fujimoto, B. Swerts, T. Miyahara, H. Nakatsuji, J. Comput. Chem. 2007, 28, 2443.

52 M. J. Frisch, Gaussian Development Version (Revision E.01), Gaussian, Inc., Pittsburgh PA, 2003.

53 R. G. Parr, W. Yang, Density-Functional Theory of Atoms and Molecules, Oxford Univ. Press, Oxford, 1989.

54 C. Lee, W. Yang, R. G. Parr, Phys. Rev. B 1988, 37, 785.

55 A. D. Becke, J. Chem. Phys. 1993, 98, 5648.

56 J. Wang, P. Cieplak, P. A. Kollman, J. Comput. Chem. 2000, 21, 1049.

57 E. Rosta, M. Klähn, A. Warshel, J. Phys. Chem. B 2006, 110, 2934.

58 T. H. Dunning, Jr., P. J. Hey, in Mehod of Electronic Structure Theory, ed. by H. F. Shaefer, III, Plenum Press, New York, 1977, p. 1.

59 H. Nakatsuji, Chem. Phys. 1983, 75, 425.

60 L. H. Andersen, I. B. Nielsen, M. B. Kristensen, M. O. A. E. Ghazaly, S. Haacke, M. B. Nielsen, M. Å. Petersen, J. Am. Chem. Soc. 2005, 127, 12347.

61 K. A. Freedman, R. S. Becker, J. Am. Chem. Soc. 1986, $108,1245$.

62 H. Houjou, Y. Inoue, M. Sakurai, J. Phys. Chem. B 2001, $105,867$.

63 M. Wanko, M. Hoffmann, T. Frauenheim, M. Elstner, J. Comput.-Aided Mol. Des. 2006, 20, 511.

64 B. Aton, A. G. Doukas, R. H. Callender, B. Becher, T. G. Ebrey, Biochemistry 1977, 16, 2995.

65 G. G. Kochendoerfer, S. W. Lin, T. P. Sakmar, R. A. Mathies, Trends Biochem. Sci. 1999, 24, 300.

66 W. Humphrey, A. Dalke, K. Schulten, J. Mol. Graphics 1996, 14, 33.

67 H. Sun, J. P. Macke, J. Nathans, Proc. Natl. Acad. Sci. U.S.A. 1997, 94, 8860.

68 A. Onishi, J. Hasegawa, H. Imai, O. Chisaka, Y. Ueda, Y. Honda, M. Tachibana, Y. Shichida, Zool. Sci. 2005, 22, 1145.

69 K. Shimono, T. Hayashi, Y. Ikeura, Y. Sudo, M. Iwamoto, N. Kamo, J. Biol. Chem. 2003, 278, 23882. 